\title{
Whole-Mount Skeletal Staining
}

\section{Diana Rigueur and Karen M. Lyons}

\begin{abstract}
The first step in almost every investigation of skeletal phenotypes is analysis of whole-mount skeletal preparations. Whole-mount skeletal staining permits evaluation of the shapes and sizes of skeletal elements in their appropriate locations. The technique is thus the major method for detecting changes in skeletal patterning. Because cartilage and bone can be distinguished by differential staining, this technique is also a powerful means to assess the pace of skeletal maturation. This protocol covers staining of the pre- and postnatal mouse skeleton using Alcian blue and Alizarin red to identify cartilage and bone, respectively.
\end{abstract}

\section{Keywords}

Alcian blue; Alizarin red; Cartilage; Bone; Cleared skeletal preparation; Whole-mount

\section{Introduction}

The formation of bone occurs through two processes: endochondral and intramembranous. Endochondral bone formation occurs after cells in mesenchymal condensations differentiate into chondrocytes. These cells secrete a cartilaginous extracellular matrix rich in proteoglycans, glycosaminoglycans (GAGs), and collagen (types II and X). Chondrocytes undergo a process of stratified differentiation and apoptosis, enabling the replacement of cartilage by bone. In contrast, during intramembranous ossification, mesenchymal cells differentiate directly into bone-forming osteoblasts. Alcian blue and Alizarin red stain cartilage and bone, respectively. As a cationic dye, Alcian blue binds strongly to sulfated GAGs and glycoproteins, while Alizarin red, an anionic dye, binds to cationic metals such as calcium [1]. Because cartilage contains higher concentrations of GAGs than any other tissue, it binds more Alcian blue. However, other tissues, such as the skin, contain GAGs and other glycoproteins that bind Alcian blue. In contrast, because $99 \%$ of the calcium in the body is localized in bone, Alizarin red is highly specific for bone. The selective staining properties of Alcian blue and Alizarin red and their use in whole-mount skeletal preparation have been widespread for over a century [2]. The protocol has been refined over the years, and there are variations employing enzymatic steps and different fixatives [2-5]. The protocol described here is used in our laboratory. Most laboratories use very similar protocols.

As a brief overview of the process, specimens are prepared by removing skin, organs, and brown fat. They are then dehydrated and fixed in $95 \%$ ethanol. For further removal of fatty tissue and tissue permeabilization, specimens are exposed to acetone and then consecutively 
transferred to Alcian blue and Alizarin red staining solutions. Concurrent with Alizarin red staining, exposure to potassium hydroxide $(\mathrm{KOH})$ hydrolyzes soft tissue, leading to transparency and allowing visualization of stained skeletal elements. The procedure can be adjusted depending on the size/age of the specimens.

\section{Materials}

\subsection{Solutions}

Use analytical grade reagents.

1. Phosphate-buffered saline (PBS): $137 \mathrm{mM} \mathrm{NaCl}, 10 \mathrm{mM}$ phosphate, $2.7 \mathrm{mM}$ $\mathrm{KCl}, \mathrm{pH}$ 7.4. To prepare $1 \mathrm{~L}$ of $1 \times \mathrm{PBS}$ pour $800 \mathrm{~mL}$ of distilled water into a beaker. Add in order $8 \mathrm{~g}$ of NaCl, $0.2 \mathrm{~g}$ of $\mathrm{KCl}, 1.44 \mathrm{~g}$ of $\mathrm{Na}_{2} \mathrm{HPO}_{4}, 0.24 \mathrm{~g}$ of $\mathrm{KH}_{2} \mathrm{PO}_{4}$. Adjust the $\mathrm{pH}$ to 7.4 with $\mathrm{HCl}$. Add distilled water to a total volume of $1 \mathrm{~L}$.

2. $100 \%$ Ethanol (EtOH).

3. $100 \%$ Acetone $\left((\mathrm{CH} 3)_{2} \mathrm{CO}\right)$.

4. Glycerol $\left(\mathrm{C}_{3} \mathrm{H}_{8} \mathrm{O}_{3}\right)$.

5. $1 \%$ Potassium hydroxide $(\mathrm{KOH}): 1 \%(\mathrm{w} / \mathrm{v}) \mathrm{KOH}, 99 \% \mathrm{dH}_{2} \mathrm{O}$. To make a 200 $\mathrm{mL}$ solution, weigh $2 \mathrm{~g}$ of $\mathrm{KOH}$ pellets. Gradually add to a beaker containing $200 \mathrm{~mL}$ of deionized water (see Note 1).

6. Alcian blue stain: $0.03 \%(\mathrm{w} / \mathrm{v}), 80 \% \mathrm{EtOH}, 20 \%$ (glacial) acetic acid. To make a $200 \mathrm{~mL}$ solution, weigh $0.06 \mathrm{~g}$ of Alcian blue 8GX and place in a beaker, add $160 \mathrm{~mL}$ of $100 \% \mathrm{EtOH}$ and $40 \mathrm{~mL}$ of $100 \%$ glacial acetic acid (see Notes 2 and $3)$.

7. Alizarin red stain: $0.005 \%(\mathrm{w} / \mathrm{v})$ in $1 \%(\mathrm{w} / \mathrm{v}) \mathrm{KOH}$. To make a $200 \mathrm{~mL}$ solution, weigh $10 \mathrm{mg}$ of Alizarin red and add to $200 \mathrm{~mL}$ of $1 \% \mathrm{KOH}$ (see Notes 4 and 5).

\subsection{Tools/Supplies}

1. Dissecting microscope and photographic equipment.

2. Diapers.

3. Hot water bath.

4. Forceps.

5. Scalpel.

\footnotetext{
${ }^{1} \mathrm{KOH}$ is highly caustic to skin. Lab coat, gloves, and goggles should be worn when working with this chemical. KOH dissolves glass, so this solution should be stored in a plastic container or made fresh.

${ }^{2}$ Mix the Alcian blue solution very well; this may take $30 \mathrm{~min}$ to an hour. Then filter the solution. Otherwise, unequal and patchy distribution of the dye will lead to uneven staining of skeletal preparations.

${ }^{3}$ This solution can be stored for several weeks but works best when freshly prepared. It should be filtered before each use.

${ }^{4}$ To stain bone a purple-red appearance, use the formula stated above. If a more orange-red appearance is preferred, you can use Alizarin red diluted in EtOH: Alizarin red: $0.05 \%$ (w/v) in $95 \%$ (w/v) EtOH. Then digest in $\mathrm{KOH}$ until specimen clears.

5 Alizarin red solutions work best when prepared freshly.
} 
6. Glass scintillation vials, $15 \mathrm{~mL}$, or $50 \mathrm{~mL}$ conical tubes.

\section{Methods}

\subsection{Staining of Skeletal Elements: Mid-Gestation Stages (E12.5-E16.5)}

1. Collect embryos following euthanization of timed pregnant females and place in $1 \times$ PBS.

2. Remove the extraembryonic membranes encircling the embryo. Remove the eyes while in PBS [3-7]. For early-stage embryos (E12.5-E14.5), it is not necessary to remove the skin or eviscerate the embryo. For older embryos, eviscerate as follows: initiate skin removal by creating a horizontal slit in the skin at the abdomen using dissecting scissors. Then pull apart using your fingers or forceps. Continue to tug the skin gently apart. Repeat by making a vertical slit in the dorsal skin of the embryo and pulling the edges apart. To remove skin from the arms, make a vertical slit on the ventral and dorsal surfaces of the fore and hind limbs. Peal the skin using forceps.

3. Place the embryos in glass scintillation vials containing $70 \%$ ethanol to fix overnight at $4{ }^{\circ} \mathrm{C}$ ( see Note 6 ).

4. Remove the $70 \% \mathrm{EtOH}$ and replace with $95 \% \mathrm{EtOH}$ for $1 \mathrm{~h}$.

5. Remove the $95 \% \mathrm{EtOH}$ and replace with acetone overnight at room temperature (see Note 7).

6. Remove the acetone and replace with Alcian blue stain for 1-4 h (see Note 8).

7. Remove the Alcian blue stain and replace with Alizarin red stain for 3-4 h (see Note 9).

8. Remove the Alizarin red stain and replace with $1 \% \mathrm{KOH}$ for $12 \mathrm{~h}$ to overnight for clearing of the embryo [5, 7-9] (see Note 10).

9. Transfer the embryos to a $50 \%$ glycerol:50\% (1\%) KOH solution at room temperature until tissue appears transparent.

10. Once cleared, transfer the specimen to $100 \%$ glycerol for long-term storage [5, 7-9]. See Fig. 1.

\footnotetext{
${ }^{6}$ The embryos can be transferred to $15 \mathrm{~mL}$ conical tubes or glass scintillation vials. We prefer the latter because it is easier to visualize the extent of staining.

${ }^{7}$ Acetone acts as a fixative, permeabilizes cell membranes to permit penetration of the stain, and dissolves fat.

${ }^{8}$ The skin of young embryos is porous enough to permit penetration of the Alcian blue stain following treatment with acetone.

However, long-term exposure to Alcian blue stain will result in a nearly irreversible over-staining of skin and other soft tissues, making it difficult to see the underlying skeletal elements. If over-staining occurs, placing the embryo in $20 \%$ glacial acetic acid can allow some leaching of the stain from soft tissues.

${ }^{9}$ Incubate the specimens in Alizarin red until the bone turns red. Over-staining will lead to irreversible uptake of the stain by muscle.

10 If the sample is kept in $\mathrm{KOH}$ too long, the embryo will disintegrate and/or skeletal elements will disarticulate. The length of time the specimen should be maintained in $\mathrm{KOH}$ depends on its size, but at the end of the incubation, the specimen should be nearly transparent.
} 


\subsection{Late-Gestation and Early-Postnatal Stages: E16.5-P21}

1. Wash and then scald the specimen in hot tap water for $20-30 \mathrm{~s}$ at $65^{\circ} \mathrm{C}$ to facilitate maceration (permeabilization) of the tissue and removal of skin [9] (see Note 11).

2. Remove eyes, all skin, internal organs, adipose tissue, and bubbles from the body cavity [7, 10] (see Notes 12 and 13).

3. Fix the embryos in $95 \%$ EtOH overnight at room temperature $[5,7-9]$.

4. Place the samples in acetone overnight at room temperature (see Note 7).

5. Stain for cartilage by submerging the embryo in a glass scintillation vial containing at least enough Alcian blue solution to cover the embryo. Incubate the sample overnight at room temperature [5, 7-9] (see Note 14).

6. Destain embryos by initially washing them in two changes of $70 \% \mathrm{EtOH}$ and then incubating them in $95 \%$ EtOH overnight [5] (see Note 15).

7. To pre-clear the tissue, remove the $95 \% \mathrm{EtOH}$ and add $1 \% \mathrm{KOH}$ solution for 1 $\mathrm{h}$ at room temperature [7].

8. Then remove the $\mathrm{KOH}$ solution, and replace it with Alizarin red solution for 3-4 $\mathrm{h}$ at room temperature. To slow down the staining, place the samples at $4{ }^{\circ} \mathrm{C}$ overnight $[5,7,9]$ ( see Note 16).

9. Replace the Alizarin red solution with a $50 \%$ glycerol:50 \% (1\%) $\mathrm{KOH}$ solution. You can incubate the specimen in this solution at room temperature or $4{ }^{\circ} \mathrm{C}$ until the excess red color is removed and the specimen is cleared [7].

10. Transfer sample in $100 \%$ glycerol for long-term storage [7-11]. See Fig. 2.

\subsection{Later Postnatal and Adult Stages (3 Weeks Old and Over)}

1. Euthanize the mouse and spray with $70 \% \mathrm{EtOH}$.

2. Remove the skin (see Subheading 3.1, step 2, and Notes 12 and 13).

3. Remove eyes and visceral organs.

4. Remove as much adipose and other excess tissue as possible (see Note 17).

\footnotetext{
${ }^{11}$ For older pups with hair, omit this step and just spray specimen generously with $70 \% \mathrm{EtOH}$ to dampen the hair to facilitate dissection.

12 One of the most commonly encountered problems with cleared skeletal preparations is insufficient staining of the digits due to failure to remove the skin around them. One way to remove the skin from hands and feet is to make a cut with a scalpel along the dorsal surface of the hand/foot and then along each finger. Then use the forceps to pinch the skin between the fingers to pull it off. ${ }^{13}$ Ensure removal of fat, organs, and bubbles from the body cavity; they impair staining by Alcian blue. It is important to remove the brown fat behind the neck and between the scapulas because it is pigmented and does not clear well. Moreover, Alcian blue stains fat. ${ }^{14}$ The entire specimen should turn blue, along with the cartilage. Weak staining can be attributed to improper evisceration, old solution, or inadequate exposure to Alcian blue. If weak staining is observed, keep the specimen in solution for a longer period of time, but this introduces a risk of over-staining.

15 Keep changing the $70 \%$ EtOH until it shows no blue coloration. This dehydration will fix the Alcian blue solution in cartilage and help destain surrounding soft tissues.

${ }_{16}$ Samples can be incubated in Alizarin red solution overnight at room temperature; however, avoid longer incubations as overstaining can occur, and the $\mathrm{KOH}$ can lead to disarticulation.
} 
5. Place in two changes of $95 \% \mathrm{EtOH}$ overnight at room temperature to dehydrate and fix the specimen.

6. Replace the solution with $100 \%$ acetone for 2 days to further fix the specimen and to remove adipose tissue (see Note 7).

7. Incubate the specimen in Alcian blue solution for 1-3 days (see Notes 8 and 14).

8. Destain embryos by initially washing them in two changes of $70 \% \mathrm{EtOH}$ and then incubating them in $95 \% \mathrm{EtOH}$ overnight $[5,7]$ ( see Note 15).

9. Replace with $1 \% \mathrm{KOH}$ for $4 \mathrm{~h}$ at room temperature to overnight at $4{ }^{\circ} \mathrm{C}$ to preclear the specimens.

10. Replace the $1 \% \mathrm{KOH}$ with Alizarin red solution, and incubate the specimens for 2-5 days (see Notes 16 and 17).

11. Transfer the specimen into $1 \% \mathrm{KOH}$ as a clearing solution (see Note 18).

12. For long-term storage, keep the specimen in $100 \%$ glycerol. See Fig. 3.

\subsection{Preparing for Imaging}

1. Carefully place the stained skeleton in a clear $60 \mathrm{~mm}$ or $10 \mathrm{~cm}$ plastic or glass dish using a scooping utensil (see Note 19).

2. Fill the plate with $100 \%$ glycerol, and allow Schlieren patterns to settle (see Note 20).

3. Place the specimen under a dissecting microscope utilizing bright field optics and a white background.

4. If necessary, trim away excess tissue (see Note 21).

\section{References}

1. Horobin, RW. How do dyes impart color to different components of the tissues? In: Kumar, GL., editor. Educational guide special stains and H \& E. 2. Carpinteria; California: 2010. p. 159-166.

2. Schultze O. Ueber herstellung und conservirung durchsichtiger embryonen zum stadium der skeletbildung. Anat Anz. 1897; 13:3-5.

3. Nagy A, Gerstsenstein M, Vintersten K, Behringer R. Alcian blue staining of the mouse fetal cartilaginous skeleton. Cold Spring Harb Protoc. 2009; doi: 10.1101/pdb.prot5169

\footnotetext{
${ }^{17}$ If the solution becomes murky, after its 2-3-day incubation, transfer the specimen to $1 \% \mathrm{KOH}$ solution. For an older mouse, $2 \%$ $\mathrm{KOH}$ exposure is acceptable; however, beware that hydrolysis is faster. Change the solution daily until no longer murky and the tissue clears. Then place in a $50 \%$ glycerol:50 \% (1\%) KOH solution for complete clearance.

${ }^{18}$ Decreasing gradients of $1 \% \mathrm{KOH}$ to glycerol can be used to further clear the specimen; however, for long-term storage, ensure that the specimen is stored in glycerol; otherwise, the $\mathrm{KOH}$ will cause the skeleton to disarticulate.

${ }^{19}$ Take a plastic disposable $1 \mathrm{~mL}$ pipette and cut off the top half of the bulb lengthwise- this creates a scoop, with the dropper forming a handle.

${ }^{20}$ The viscosity of glycerol facilitates positioning of the skeleton for imaging; however, Schlieren patterns result from differences in solution concentrations of glycerol or salt content. It can take several hours to overnight for these to disappear. Storing the specimens in a large enough volume of $100 \%$ glycerol or graded concentrations of glycerol to EtOH, to completely submerge the specimen when the contents of the vial are transferred to the dish will eliminate this problem.

${ }^{21}$ Removing excess tissue at this stage makes a big difference to the final quality of the image. For larger embryos and postnatal stages, forceps may be sufficient. For mid-gestation embryos, a set of 4-in. straight micro scissors is an excellent investment.
} 
4. Jegalian BC, De Robertis EM. Homeotic transformations in the mouse induced by over expression of a human Hox3.3 transgene. Cell. 1992; 71:901-910. [PubMed: 1360874]

5. Estrada K, Retting KN, Chin AM, et al. Smad6 is essential to limit bmp signaling during cartilage development. J Bone Miner Res. 2011; 26(10):2498-2510. [PubMed: 21681813]

6. Nagy A, Gerstsenstein M, Vintersten K, Behringer R. Isolating extraembryonic membranes. Cold Spring Harb Protoc. 2006; doi: 10.1101/pdb.prot4267

7. Hogan, BLM., Beddington, R., Costantini, F., Lacy, E. Manipulating the mouse embryo: a laboratory manual. Cold Spring Harbor Laboratory Press; Cold Spring Harbor, NY: 1994.

8. Retting KN, Song B, Yoon BS, Lyons KM. BMP and Smad signaling through Smad1 and Smad5 is required for endochondral bone formation. Development. 2009; 136(7):1093-1104. [PubMed: 19224984]

9. Ovchinnikov D. Alcian blue/Alizarin red staining of cartilage and bone in mouse. Cold Spring Harb Protoc. 2009; doi: 10.1101/pdbprot5170

10. Nagy A, Gerstsenstein M, Vintersten K, Behringer R. Alizarin red staining of post-natal bone in mouse. Cold Spring Harb Protoc. 2009; doi: 10.1101/pdb.prot5171

11. Green MC. A rapid method for clearing and staining specimens for the demonstration of bone. Ohio J Sci. 1952; 52(1):31-33. 
a

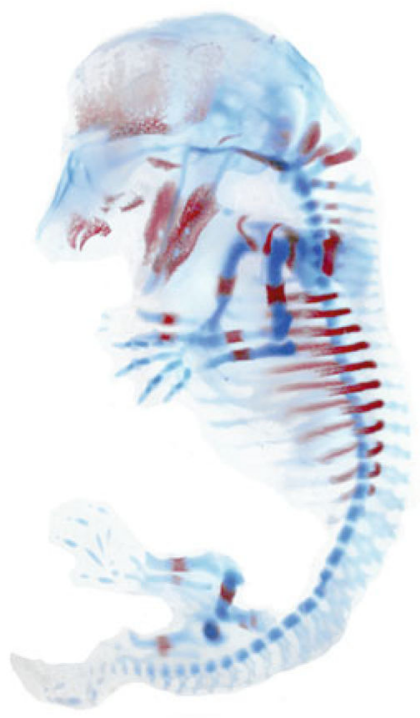

b

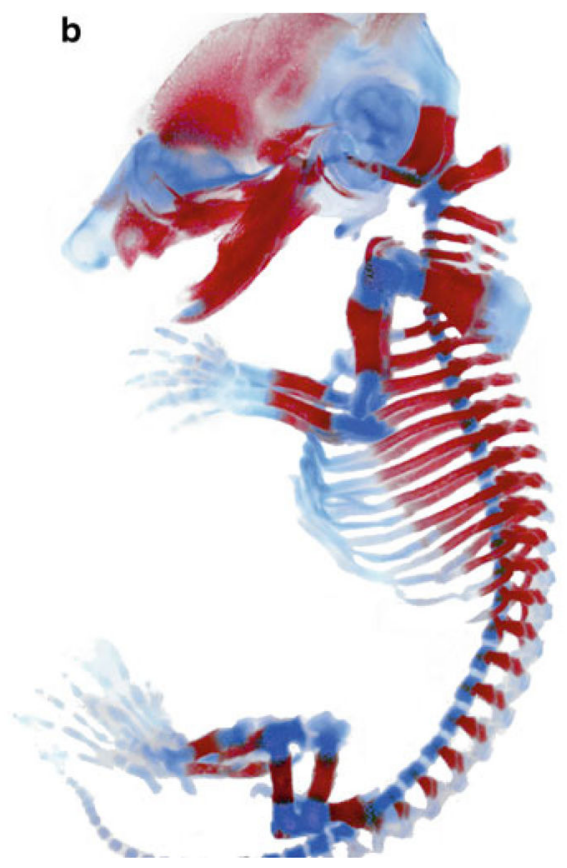

Fig. 1.

Alcian blue and Alizarin red staining of (a) E14.5 and (b) E16.5 embryos. Images were taken using bright field optics. Following adjustment of color levels using Photoshop, the embryos were pasted onto a white background 


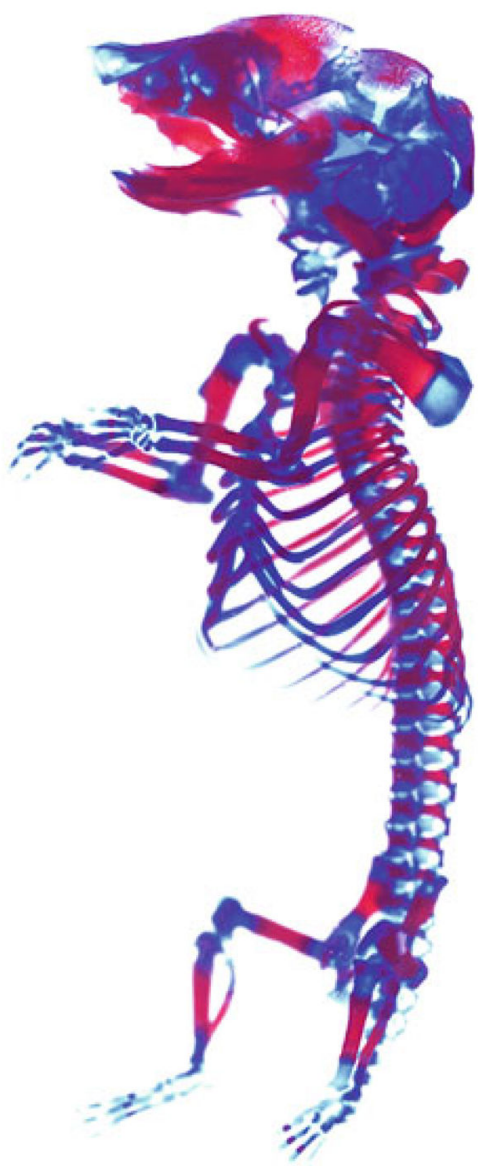

Fig. 2.

Alcian blue and Alizarin red staining of a P0 pup. Image was processed as in Fig. 1 


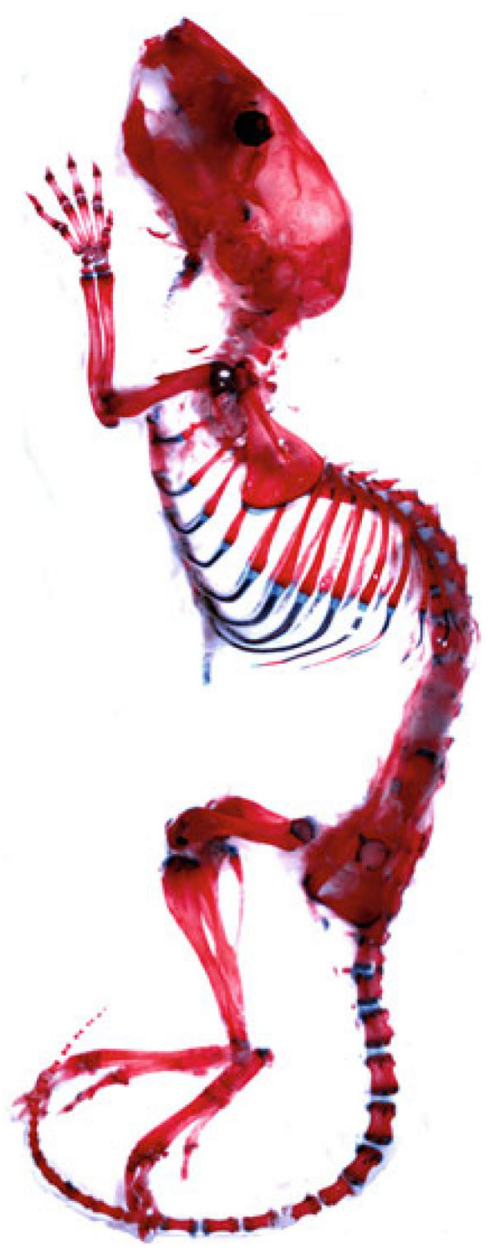

Fig. 3.

Alcian blue and Alizarin red staining of a 4-week-old postnatal mouse. The image was processed as in Fig. 1 